\title{
Semantics of Empirical Research and Its Pedagogic Application
}

\author{
Lisheng Huang ${ }^{1, \#}$, Mingqi Wang, ${ }^{2, \#}$, Yingyu Cui ${ }^{3, *}$ \\ ${ }^{1}$ Tongji University school of law, Shanghai, China \\ ${ }^{2}$ Tongji University School of electronic information engineering, Shanghai, China \\ ${ }^{3}$ Department of Cell Biology, Tongji University School of Medicine, Shanghai, China \\ \#These authors contributed equally to this work and share first authorship \\ *Corresponding author. Email: yycui@tongji.edu.cn
}

\begin{abstract}
Purposes: To retrospect the origin of empirical research and probe into its semantics. Procedures: Retrieving, sorting, reading and analysing literatures, then trying to summarize the history of empirical research and its pedagogic application. Methods: CNKI, Wan-Fang, and VIP databases, etc. were used to search for the related literatures, and MindMaster 8.5.0 software was used to draw the map of empirical research methods. Results: The origin of empirical research and its impact on pedagogy were summarized, and the corresponding methods were compared and evaluated, which provided guidance for the further research of empirical research in pedagogy. Conclusions: Clarification of the semantics of empirical research and its organic introduction into traditional pedagogy research enhanced the validity, scientificity and credibility of pedagogical research results.
\end{abstract}

Keywords: Research-based learning, Empirical research, Pedagogy, Research skills.

\section{SEMANTICS OF EMPIRICAL RESEARCH}

Empirical research is a novel research method developed and perfected under the guidance of positivism philosophy. Therefore, to understand its essence, we need to go back to the source of positivist philosophy. The system of positivist philosophy originated from the Industrial Revolution. People gradually formed a new world outlook and methodology, among which a very important product is positivism.

The earliest representatives of positivism include Auguste Comte, John Stuart Mill, Herbert Spencer, et al. They made respective achievements in the field of positivism philosophy, but they all held common basic viewpoints, in detail, they all attached importance to the key role of empirical facts for laws and results; they all strongly opposed metaphysical philosophy. Metaphysics emphasizes the exploration and discovery of the causality and origin of all existing things, and also pays attention to the free will of human being.

The Post-positivism has also been inherited and developed. For example, Machism in the 1870s and the Vienna School in the 1920s are the third generation of positivism with characteristics of logical positivism and logical empiricism. [1]

Positivism philosophy is a main branch of philosophy. Here, we only try to show its development and evolution, further laid the basis for the empirical research.

The early positivist philosophers paid more attention to the situation of society, and exploring solutions to the related social problems. Interactive methodology and practical guidance objectively promoted positivism to effectively guide the development of social sciences (mainly sociology). At this stage, positivism had not been completely developed into a philosophical system and a perfect social science research method.

As for the idea of positivism integration into the social sciences and becoming a systematic methodology, we believe that it is an issue varying with special circumstances and may also be widely disputed in the academic fields. Under the influence of positivism philosophy, positivism as a research thought has gradually permeated into some studies of sociology. After that, with the other major branches of social sciences' infiltration, sociology developed a complete system, but there were certain temporal and spatial 
differences among them. When it has become common practice as a scientific research method in the other countries, Chinese educational scholars paid more attention to logical speculation and the traditional research methods. "Empirical research papers of pedagogy in our country account for less than $15 \%$, and most are still in the stage of subjective speculation and contingent imagination." [2]

Empirical research actually means that positivist researchers put forward their own theoretical propositions through a large number of systematic observations, empirical verifications, or investigation, analysis and comparison, and realize the value effect of relevant conclusions from the perspective of improving the validity of the research from individual to general. There are three empirical research methods: confirming facts and experience, discovering the similarity of phenomena and establishing theoretical regularity of phenomena, testing the development of theory with practice.[3]

After clarifying the concept of empirical research, we need to have a general grasp of the connotation extension of empirical research. What kind of research can be called empirical research, and what tools can be used by empirical researchers?

\section{THE THEORETICAL BASIS OF EMPIRICAL RESEARCH}

Empirical research is a certainly powerful tool for social science research. Firstly, we should understand the scope of social science research and empirical research methods. Only this way, can we make full use of these methods and tools to serve a topic of research. For this purpose, we drew the following framework of social science research methods (Figure.1) with emphasis on the first part.

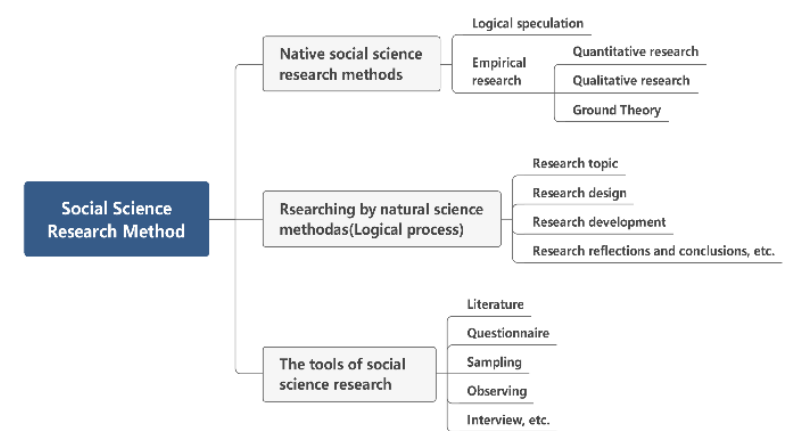

Figure 1 Methods to Social Science Research

In some fields, the academic circles have not reached a unanimous point of view. For example, many scholars think that qualitative research and quantitative research are two completely different research methods, even of absolute contradiction. However, some scholars refute, and insist that as long as a research is based on facts and evidence, it is empirical research, regardless of whether it is quantitative or qualitative analysis.[4]
In addition, whether qualitative research belongs to empirical research is another key issue. Truly, qualitative research really emphasizes that social science research is a process of knowledge construction under natural circumstances. And this paradigm of hermeneutics or constructivism methodology emphasizes the subjective position. [5]

Although the three main methods are quite different, the key to distinguishing whether it can be regarded as an empirical research is whether it has indeed been verified by the researchers' long-term systematic investigation and case verification or falsification. From this perspective, the three can be well applied to this point. Although many people believe that the qualitative research of constructivism has no pre-research expectations, we insist that every research topic must respond to what the research is. Therefore, the so-called qualitative research of knowledge construction also has traces to follow.

Many domestic educators have begun to pay attention to this problem. Many authors agree with the point that the future of educational technology research is not to judge the choice between behaviorism, cognitivism, constructivism and situationalism, but to explore what kind of teaching theory is suitable for guiding students and developing their thinking capabilities.[6]

\subsection{Qualitative Research}

Qualitative research emphasizes the exploration of causal mechanism. The so-called causal mechanism is to realize a deep description and explanation of the mechanism between independent variables and dependent variables in a small sample environment. In fact, the greatest value of qualitative research itself is often to present some of the interaction processes between the two variables that have been proposed as having a causal relationship. Through in-depth, meticulous and long-term experience, investigation and analysis to the interactive relationship, qualitative research obtains a more comprehensive and profound understanding of things.[7] Compared with quantitative research, the shortcoming of qualitative research is that it may not have strong external validity. The so-called external validity refers to the extent to which relevant conclusions are promoted to enrich the theory.

Even such a descriptive exploration can achieve depth that other research methods do not have. In addition, it does not completely reject quantitative thinking and research tools. For example, many educational scholars have criticized learning-weary students. Questionnaire surveys are also qualitative research. Questions related to qualitative research are often small samples, such as open questionnaires that are carried out for one or a few individuals, which are 
helpful for research, because open questionnaires can often deepen the process, and the corresponding description may play a very good role.

\subsection{Quantitative Study}

Quantitative research is often relative to qualitative research. Quantitative research focuses on whether the dependent variable and independent variable in the research topic really exist in this causal effect. In other words, quantitative research itself is used to resolve the two that originally existed in a superficial correlation, and to explain whether there is a causal effect. As an example cited by Liu, there is a positive correlation between the increase in the number of drowning on the beach in summer and the increase in ice cream sales. But in fact, there is no causal relationship between them, because hot weather causes the number of swimmers and people buying ice cream both increased. [8] This case also proves that quantitative research is easy to ignore the process of things, and may result in fake causal relationship.

\subsection{Ground Theory}

Ground theory requires the researchers to use a variety of data collection methods in a natural context to conduct in-depth and holistic research on some phenomenon, to draw conclusions and put forward theories from the original data, and to interact with the research objectives to determine their behaviors and meaning construction, in other words, it demands a series of activities to gain explanatory understanding of the phenomenon. [9] The biggest difference is that qualitative research emphasizes the construction of knowledge, and ground theory emphasizes the construction process based on the interaction between researchers and those needing humanistic care, rather than isolated from the situation and maintain a neutral attitude. Obviously, ground theory is necessary for the creation of new theories during the research process, therefore, easier to achieve theoretical innovation.

Summarily, the above methods need to be used in a reasonable and timely manner during the process of social sciences research. Reasonable use of them can effectively strengthen the validity of research conclusions, and further improve the efficiency and quality of research.

\section{EMPIRICAL RESEARCH IN PEDAGOGY}

Research-based learning (RBL) is a dark-horse in the fields of pedagogy, or a multifaceted approach for connecting research and instruction, integrating learning, teaching and research. Its central idea is to actively involve students in on-going research activities and let them develop their own research interests and questions [10]. However, empirical researches were not fully utilized to support its significance in higher education. And the academic community just roughly formed a certain theoretical consensus on RBL. For example, Li believed RBL refers to a teaching method in which students conduct active exploration by selecting certain topics themselves, [11] while Zhang thought RBL refers to the pathway students acquire knowledge and apply knowledge through scientific research under the guidance of teachers. [12]

Summarizing the characteristics of RBL from literatures of relevant scholars, RBL is an autonomous and exploratory learning style under the guidance of teachers, emphasizing the process of experiencing, learning and acquiring knowledge. In short, the professional learning that college students are engaged in can be regarded as a branch of RBL, but since college students' professional knowledge is just beginning and limited, we try our best to help those who come across $\mathrm{RBL}$ for the first time through teaching reforms.

\subsection{Taking Advantage of Ready Methods}

The three research methods can complement each other in some respects. With the advancement of research content, we can use qualitative methods to conduct in-depth qualitative research on key content nodes, such as some of the research learners' online and offline mixed teaching and the final learning results under the restrictions of this research. We also use a combination of quantitative research methods such as questionnaires, scales, and literature to explore whether our learning participants have really improved their critical and innovative thinking in the process under such an interactive environment, and feel how the learning mode full of humanistic care enables learners and teachers to build novel theory together.

\subsection{Enriching Pedagogy Theory}

Our research will enhance the enrichment of pedagogy theory through the interaction between teachers and students and the corresponding testing results from both. The promotion of external validity and the extension of conclusions should be paid attention to what factors affect the results of teaching and learning, further to what extent these factors have changed education itself. The research quality demands us to read a lot of literature, further quantitatively measure related concepts, and to ensure the process scientific and reasonable, and we expect to make an effective supplement to some areas that others have not probed into at all.

\section{CONCLUSION}

Some advantages of the ready methods, also the foothold of empirical research on RBL were 
summarized here. In fact, it has long been a consensus in the academic field that pedagogy should adopt empirical research, or a hybrid research of certain methods in empirical research fields. For example, Yao pointed out that "my country's educational research methods should pay emphasis on the construction of systems and standards; strengthen the empirical research orientation and promote the diversification of research paradigms; integrate quantitative and qualitative research methods and explore hybrid research." [13]

Frankly, we should admit that empirical studies will ignore some humanistic concerns, but we can also use educational experiments to make up for it and obtain detailed first-hand data; we know that social science research, especially pedagogy research, is different from traditional natural sciences, but different disciplines and methods, the intersection of pedagogy really make the content of pedagogy research deeper and more advanced.

\section{ACKNOWLEDGMENT}

The project is partly supported by Tongji University Undergraduate Innovation and Entrepreneurship Training Program Project: "Empirical Research in Online, Offline and Mixed Teaching of Research-based Learning” (No. X202014009) and Shanghai Educational Research Project (No. C19154 to YY Cui).

\section{REFERENCES}

[1] Jun Li, On the Paradigm of Positivism and Its Significance to Pedagogy, Educational Research, 2018, (7):41-48.

[2] Zhenguo Yuan, Empirical research is a necessary way for pedagogy to move towards, Journal of East China Normal University (Education Science Edition), 2017, (3):4-17.

[3] Qingxiong Zhang, Philosophy of Social SciencesPositivism, Hermeneutics and Wittgenstein's Transformation Fudan University press, 2018, (1): 12.

[4] Zhenguo Yuan, Empirical research is a necessary way for pedagogy to move towards, Journal of East China Normal University (Education Science Edition), 2017, (3):4-17.

[5] Zhiyong Zhu, Reflection on the Paradigm and Method of Educational Research Methodology, Educational Research and Experiment, 2015, (1):712.

[6] Xiaodong Xu, Postmodern Educational Technology Research Method and Methodology, Open Education Research, 2003, (4):12-15.
[7] Xiangming Cheng, A Review of Qualitative Research Methods, Educational Research and Experiment, 1996, (3):62-68.

[8] Zhilin Lin, The social methods, [EB/OL] https://www.xuetangx.com/learn/THU0303100066 6, 2020-7-15.

[9] Xiaoying Lin, Qualitative research methods,[EB/OL]https://www.xuetangx.com/learn/ PKU04011000020/PKU04011000020/4227207/vid eo/6192004, 2020-7-15.

[10] Yingyu Cui, Jun Zhang, Yihan Chen. ResearchBased Learning Remodels the Characters of Undergraduates for Creative and Innovative Personnel. Advances in Social Science, Education and Humanities Research 2019; 361:76-80

[11] Shaocun Li, Probing into Research Study, Chinese Journal of Education, 2001, (1):52-54.

[12] Zhaofeng Zhang, On Research Study. Curriculum Teaching Material and Method, 2000, (6):42-45.

[13] Jihai Yao, Paradigm Questions and Reflections on Educational Empirical Research Methods, Journal of East China Normal University, 2017, (3):64-71. 